\title{
Maximus: a Hybrid Particle-in-Cell Code for Microscopic Modeling of Collisionless Plasmas
}

\author{
J.A. Kropotina ${ }^{1,2}$, A.M. Bykov ${ }^{1,2}$, \\ A.M. Krassilchtchikov ${ }^{1}$, and K.P. Levenfish ${ }^{1}$ \\ ${ }^{1}$ Ioffe Institute, St. Petersburg, Russia \\ ${ }^{2}$ Peter the Great St. Petersburg Polytechnic University, St. Petersburg, Russia \\ email: juliett.k@gmail.com
}

\begin{abstract}
A second-order accurate divergence-conserving hybrid particlein-cell code Maximus has been developed for microscopic modeling of collisionless plasmas. The main specifics of the code include a constrained transport algorithm for exact conservation of magnetic field divergence, a Boris-type particle pusher, a weighted particle momentum deposit on the cells of the $3 \mathrm{~d}$ spatial grid, an ability to model multispecies plasmas, and an adaptive time step. The code is efficiently parallelized for running on supercomputers by means of the message passing interface (MPI) technology; an analysis of parallelization efficiency and overall resource intensity is presented. A Maximus simulation of the shocked flow in the Solar wind is shown to agree well with the observations of the Ion Release Module (IRM) aboard the Active Magnetospheric Particle Tracer Explorers interplanetary mission.
\end{abstract}

Keywords: hybrid particle-in-cell modeling · high-performance computing $\cdot$ shocked collisionless plasmas $\cdot$ the Solar wind

\section{Introduction}

Collisionless plasmas are ubiquitous in space on a vast range of scales from the Earth magnetosphere to galaxy clusters - the largest bound objects in the universe. The growing amount and enhancing quality of multiwavelength observational data require adequate models of the observed objects and structures to be developed and confronted with the data. The required modeling is a very complicated task, as it has to account with sufficient accuracy and detalization for physical processes occurring on a very broad range of spatial and temporal scales, such as self-consistent acceleration of charged particles along with the evolution of the underlying bulk plasma flows and multiscale electromagnetic fields.

As substantial energy of the bulk flows can be converted into accelerated particles, whose dynamical role and back-reaction on the structure of the flows is significant, the modeling can not be performed within the frame of magnetic 
hydrodynamics - it has to be done on a microscopic kinetic level, and even further, within a particle-in-cell approach, where dynamics of individual particles (ions and electrons) is modelled on a spatial grid with piecewise-constant values of the electromagnetic field.

Hybrid models are a special class of particle-in-cell models of plasma flows, where the ions are treated as individual particles, while the electrons are considered as a collisionless massless fluid [1/2]3. Such an approach allows one to resolve nonlinear collisionless effects on ion scales, at the same time saving substantial computational resources due to gyro-averaging of fast small-scale motions of the electrons. Hybrid codes are employed to study various astrophysical processes, such as evolution of ion-ion instabilities or shocked collisionless plasma flows $4|5| 6|7| 8 \mid 910$, with the advantage of much greater time and spatial scales compared to those of the full particle-in-cell approach.

Here we present a detailed description of the numerical scheme and parallelization technique of Maximu $\$$, a second-order accurate divergence-conserving hybrid particle-in-cell code intended for modeling of nonlinear evolution of collisionless plasma flows with shocks. Previous versions of the code are briefly described in [11]12. The main features of Maximus are: a divergence-conserving constrained transport approach based on finite-volume discretization, a linearized Riemann solver with parabolic reconstruction, an ability to treat multi-species flows, a Boris-type particle pusher. In the new version of the code, the numerical scheme and parallelization technique are advanced, allowing for better performance, stability, and energy conservation. The recent improvements include:

(i) an account for the non-zero electron pressure;

(ii) an advanced particle pusher with time-centered electromagnetic fields (instead of previously used "current advance method" [1 combined with a simple particle mover based on Taylor expansion);

(iii) a triangular-shaped cloud (TSC) particle model used instead of the nearestgrid point (NGP) model for charge deposit and force interpolation [13]14;

(iii) $2 \mathrm{~d}$ parallelization in physical space with adaptive sizes of areas, assigned to particular MPI processes;

(iv) adaptive time steps.

The structure of the paper is as follows. Equations governing dynamics of the ions and the electromagnetic field are discussed in Section 2 The overall numerical approach is outlined in Section 3 and briefly compared with the approaches of codes dHybrid [15] and Pegasus [16. The technique and efficiency of code parallelization is illustrated in Section 3.4 where some estimates of computational resources needed to reach the scales required for typical astrophysical applications are given. It is shown in Section 5 how Maximus can be used for modeling of particle acceleration in the Solar wind. The simulated particle spectra agree with in-situ measurements of the AMPTE/IRM interplanetary mission [17].

3 The code is named after out late colleague, Maxim Gustov (1978-2014), who developed its first version in 2005. 


\section{Dymanics of the Ions and Evolution of the Electromagnetic Field}

A standard set of equations used for hybrid modeling of ion dynamics and evolution of the electromagnetic field (see, e.g., [1]) can be formulated as follows:

$$
\begin{aligned}
& \frac{d \boldsymbol{r}_{k}}{d t}=\boldsymbol{V}_{k} \\
& \frac{d \boldsymbol{V}_{k}}{d t}=\frac{Z_{k}}{m_{k}}\left(\boldsymbol{E}+\boldsymbol{V}_{k} \times \boldsymbol{B}\right) \\
& \frac{\partial \boldsymbol{B}}{\partial t}=-\nabla \times \boldsymbol{E} \\
& \boldsymbol{E}=\frac{1}{\rho_{c}}(\nabla \times \boldsymbol{B}) \times \boldsymbol{B}-\frac{1}{\rho_{c}}\left(\boldsymbol{j}_{\text {ions }} \times \boldsymbol{B}\right)-\nabla P_{e} / \rho_{c} \\
& \boldsymbol{j}_{\text {ions }}=\sum_{\text {cell }} S\left(\boldsymbol{r}_{k}\right) Z_{k} V_{k}, \quad \rho_{c}=\sum_{\text {cell }} S\left(\boldsymbol{r}_{k}\right) Z_{k}
\end{aligned}
$$

Here $\boldsymbol{r}_{k}$ and $\boldsymbol{V}_{k}, Z_{k}$ and $m_{k}$ are positions, velocities, charges, and masses of individual ions, $\boldsymbol{E}, \boldsymbol{B}$ denote the electric and magnetic field vectors, $\rho_{c}$ and $j_{c}$ are the ion charge and the density of the electric current integrated over a cell of the numerical grid. The latter are derived from positions of the ions via a weighting function $S\left(\boldsymbol{r}_{k}\right)$. The electron pressure $P_{e}$ is derived from an isothermal gyrotropic Maxwellian (this can be justified by expanding the electric VlasovLandau kinetic equation by the square root of the electron-to-ion mass ratio [18]). Similar treatment of the electrons is employed in Pegasus. In the early versions of $d H y b r i d$ the electron pressure is neglected, later versions include it in the adiabatic limit 7 . Actually, both the adiabatic and isothermal approaches are somewhat artificial and seem to have little impact on the ion scales. Some recent developments consider finite-mass electrons [19, which is more physical and will be implemented in Maximus mainly for stability reasons.

The generalized Ohm's law (equation 4) can be represented as:

$$
E_{j}=-\frac{1}{\rho_{c}} \frac{\partial P_{i j}}{\partial x_{i}}-\frac{1}{\rho_{c}}\left(\boldsymbol{j}_{\text {ions }} \times \boldsymbol{B}\right)_{j},
$$

where $P_{i j}=\left(B^{2} / 2+P_{e}\right) \delta_{i j}-B_{i} B_{j}$ is the pressure tensor.

These equations are further normalized by a number of natural scale units: the proton inertial length $l_{i}=c \sqrt{m_{p} / 4 \pi n_{0} e^{2}}$, the strength of the unperturbed large-scale magnetic field $B_{0}$, the inverse proton gyrofrequency $\Omega=e B_{0} / m_{p} c$, and the Alfven speed $V_{A}=B_{0}\left(4 \pi \rho_{0}\right)^{-1 / 2}\left(n_{0}\right.$ and $\rho_{0}$ are the unperturbed plasma number and mass density respectively 4$)$.

\footnotetext{
4 Maximus allows treatment of multiple sorts of ions. However, the normalization units are always defined for the "reference" pure hydrogen plasma of the same initial mass density $\rho_{0}$ as the particular modeled composition.
} 


\section{The Numerical Approach}

To model evolution of the ions from some initial state in a predefined spatial area, equations (15) are numerically solved (advanced in time) on a threedimensional Cartesian grid via the standard loop of procedures, which consists of the following steps:

1. Moment Collector (calculate charges and currents from equations 5)

2. Field Solver (solve equations 3 and 4 to follow the evolution of the electromagnetic field)

3. Particle Mover (solve equations 1 and 2 to move individual ions through the grid cells)

These steps are described in more detail below.

\subsection{Moment Collector}

At the Moment Collector step the velocities and positions of all particles are used to calculate charge and current densities in each of the grid cells.

According to the standard technique of particle-in-cell modeling, a "macroparticle" approximation is used, with the macro-particles occupying certain areas in the discrete phase space, i.e., representing an ensemble of real ("physical") particles, which move together. This approximation is inevitable due to a huge difference between the number of particles in real physical systems and the maximum number of simulated particles allowed by the available computational resources. The main reason to use the finite-sized macro-particles with charge and mass distributed over multiple cells is suppression of the strong numerical noise caused by the limited number of particles per grid cell ( $p p c)$. The shapes of the macro-particles are defined by the weighting function $S\left(\boldsymbol{r}_{k}\right)$ (see equations 5 ). The impact of a weighting function on characteristic collision and heating time was studied by [14. Compared to the case of point-like particles, with a secondorder accurate triangular-shaped-cloud (TSC) weighting function we achieve an order of magnitude improvement of energy and momentum conservation, which could not be achieved by any reasonable increase of ppc. On the other hand, finite-sized particles can suppress the instabilities with wavelengths less than the particle size. However, the TSC length is only twice that of a cell and $75 \%$ of its weight is contained within one cell length, so the minimal wavelengths are still close to the grid resolution. Similar weighting is employed in the hybrid codes dHybrid and Pegasus. The TSC function is used to deposit charge and current densities (hereafter "cell moments") onto the mesh. The cell moments are further used to update the values of the electromagnetic field at the Field Solver step of the algorithm.

\section{$3.2 \quad$ Field Solver}

In order to keep the divergence of magnetic field vector equal to zero, a Godunovtype constrained transport scheme 20] has been implemented. It employs the 
staggered grid with edge-centered edge-aligned electric fields and face-centered face-normal magnetic fields. Additional time splitting is used to simultaneously solve the interconnected equations 3 and 4, Advancing physical time (with cell moments kept constant) requires the values of electric and magnetic field to be leap-frogged several times in the following way:

1. Spatial derivatives of the face-centered values of the magnetic field are found. In order to keep the total variation diminishing (TVD) condition, the monotonized central-difference limiter is used at this step (see, e.g., [21]).

2. A piecewise-parabolic reconstruction of magnetic field inside each cell is made, employing the values of the normal component of $\boldsymbol{B}$ and their spatial derivatives. The reconstruction coefficients are calculated according to 20].

3. A linearized Riemann solver [22] is used to obtain $\boldsymbol{E}$ on the cell edges from equation 4

4. The face-centered magnetic field is updated from equation 3 .

In the middle of this step the time- and cell-centered values of $\boldsymbol{E}$ and $\boldsymbol{B}$ are obtained, to be further used at the Particle Mover step. For this the face-centered values of the pressure tensor $P_{i j}$ are calculated with a linearized Riemann solver near the cell faces. The electric field in the cell center is then found from equation 6] via a numerical differentiation of $P_{i j}$. At the same time, the magnetic field is found via cell-averaging of the current parabolic reconstruction. Compared to Maximus, dHybrid seems to lack a constrained transport algorithm, while in Pegasus the cell-centered values of the magnetic field are simple face averages, and the evaluation of edge electric fields is not specified [16].

\subsection{Particle Mover}

A Boris-type particle pusher [13|2] is used to propagate the ions. The electromagnetic field at each particle position is calculated by interpolation of timeand cell-centered field values with the same weighting function as during the Moment Collector step. This ensures the second order accuracy in time and space together with the absence of self-forces. To increase the accuracy, the following predictor-corrector algorithm is used:

1. The "initial" Lorentz force is calculated from current particle positions and velocities using field interpolation.

2. The "predicted" particle positions and velocities at half time-step are obtained from equations 1 and 2

3. The "predicted" Lorentz force is found from "predicted" particle positions and velocities.

4. The particles are moved from the "predicted" to final positions using the "predicted" Lorentz force. 


\subsection{Implementation}

The code is written in $\mathrm{C}++$. The following structure types are used: PARTICLE, SORT, CELL, FACE, EDGE, VERTEX. As particles are extremely memoryconsuming due to their large number, each PARTICLE structure contains only coordinates and velocity components, but neither charge, nor mass. Particles of the same sort are organized in lists inside each cell of the grid, so that they are promptly accessible. Their charge, mass, and abundance are stored only once in a separate SORT structure. Cell-centered field values and cell moments are stored in CELLs, while FACEs contain values of $\boldsymbol{B}$ normal components and $P_{i j}$ components, EDGEs contain the values of the electric field. VERTEXes are used to store some temporary intermediate values, which are used at the Field Solver step.

The numerical scheme of Maximus is illustrated in figure 1. All the variables are divided into 5 color-coded categories, corresponding to the structures mentioned above. CELLs moments and fields are separated for clarity.

Each time step starts at $t^{n}$ with known particle positions and normal components of field vectors. First cell moments for the same moment of time are calculated at the Moment Collector step (substep 1). Then edges of $\boldsymbol{E}$ and faces of $\boldsymbol{B}$ are leap-frogged to half-timestep via the constrained transport algorithm (substep 2.1). Next, the same algorithm is used to find the half-timestep pressure tensor at cell faces (substep 2.2) and the cell-centered fields are stored (substep 2.3). Then the edges of $\boldsymbol{E}$ and the faces of $\boldsymbol{B}$ are leap-frogged until the end of the time step (substep2.4). Finally, the particles are moved to their new positions (substep 3), and the next step $t^{n+1}$ starts. Due to a similar field solver, the scheme of Pegasus is much the same, though Maximus takes the advantage of just one Particle Mover and Moment Collector per step, at the same time keeping the time-centering scheme.

The size of a time step can be automatically adjusted to comply with the numerical stability criteria [15] and the Courant condition, for the particles not to cross more than one cell during one time step. Such an adaptive time step seems to be surprisingly absent in other publicly known hybrid codes, though it can lead to a substantial speed-up of the computations.

The program sequence is realized by means of a sorted list, which allows one to flexibly change the set of procedures (for example, switch off the Moment Collector and Field Solver to study test particle movement in external fields).

\section{Parallelization technique and efficiency}

Usual tasks for hybrid simulations are highly resource-intensive, as the modelled systems must be resolved on scales of ion inertial lengths and time periods less than their inverse gyrofrequencies. At the same time, sizes and lifetimes of typical astrophysical objects, like supernova remnants, are many orders of magnitude larger. Hence hybrid simulations of such objects as a whole are not realistic. Even though only a tiny part of the real object is modelled, its size 
and modeling time still must be substantial to study long-wave, slowly growing instabilities, transport of energetic particles, and their back-reaction on the background plasma.

Another crucial parameter is the $p p c$ number. The numerical noise of the electromagnetic field values, which is due to the limited number of macro-particles, typically scales as $\delta B / B \sim p p c^{-1 / 2}$. Hence $p p c$ typically has to exceed 100 to guarantee the accuracy of simulation at least at the $10 \%$ level. Simulation of some finer effects may demand the ppc to exceed several thousand ([23|24|25]). The effects of limited $p p c$ on simulations of astrophysical shocks were investigated in [12. It should be noted that sometimes hybrid simulations of space plasmas are performed at $p p c=8$ or even $p p c=4$ [15/7. However, such simulations obviously require an artificial suppression of the numerical noise via low-pass filtering. Great care should be taken in such cases, as conservation of energy and spectra of the electromagnetic field are likely violated.

Due to their high resource-intensity, hybrid codes can be efficiently run only on multi-core computers and clusters. The Message Passing Interface (MPI) parallelization technology was chosen for Maximus: different MPI processes operate on different spatial parts of the simulation box. Various tests have shown that the inclusion of the third spatial dimension is usually less important for the modelled physical systems than maintaining substantial simulation box sizes in $2 \mathrm{~d}$. So Maximus is usually run within $2 \mathrm{~d}$ boxes, though all the three components of velocity and field vectors are considered. Hence, the division of the modelled physical area between the MPI processes is done in two dimensions.

The directions of such division are automatically chosen along the two maximum simulation box sizes for each particular run. The number of processes per each direction is adjusted so that each area is made square-like. Notably, when modeling a shock, the area of $r$ times higher density (i.e., higher $p p c$ ) appears downstream ( $r$ is the shock compression, which equals 4 for a typical strong shock in a monoatomic gas). Hence, the MPI regions in the shock downstream are made 4 times smaller to balance the load. When modeled shock is launched at $t=0$ and then moves across the simulation box, the downstream region grows with time. In this case the processes domains are apriori made 4 times smaller in the region, which will be in downstream at the end of simulation.

The numerical scheme assumes that each cell influences not only its 26 closest 3d neighbours (for implementation of TSC particles), but also the neighbours of neighbours (to interpolate forces at the predicted positions of particles during the Particle Mover step, see Section (3). So the spatial region of each process is surrounded by two layers of ghost cells, used for processes communication (see Fig. 21). One layer of ghost faces is also introduced for the Field Solver step. It should be noted that in the case of small spatial regions number of ghost cells exceeds number of cells inside the domain, and memory and time for communication become relatively significant. However, this is usually not the case in large astrophysical setups.

In order to access the parallelization efficiency, an illustrative setup of Maximus was run on several sets of processes (from 1 to $28 \times 8=224$ ) on the "Tornado" 
cluster of SPbPU, which has 612 28-core nodes, each with $64 \mathrm{~Gb}$ RAM and $2 \mathrm{x}$ Intel Xeon CPU E5-2697 v3 (2.60GHz). The setup was chosen large enough to eliminate relative communication expenses. Also the box size was taken to be divisible by the number of cores per node, so that the processes treated equal space regions. In total, 28000 spatial cells were modelled with single sort $p p c$ $=100$ and periodic boundary conditions applied for particles and fields. The Moment Collector, Field Solver, and Particle Mover procedures were executed with fixed time step $d t=0.005 \Omega^{-1}$ until $t=1000 \Omega^{-1}$ (i.e., 200000 time steps were made). Two simulation box shapes were chosen: $1 \mathrm{~d}(28000 \times 1 \times 1$ cells $)$ and $2 \mathrm{~d}(280 \times 1 \times 100$ cells $)$.

Fig. 22 shows the decrease of total run time with the increase of the processes number. One can see that the run time scales well with the number of processes, even if more than one node is employed. This indicates the relatively small contribution of internode communications to the total resource consumption, which is expected for large box sizes. Similar estimates for dHybrid [15] (with box size of 96 cells) showed substantial communication expenses due to small domain sizes. The difference between the $1 \mathrm{~d}$ and $2 \mathrm{~d}$ configurations is not substantial, though 1d appears slightly quicker. Overall, the characteristic run time per particle per step is almost constant and can be estimated as $t_{p s} \sim 500 \mathrm{~ns}$.

Consider a typical problem of high energy astrophysics - simulation of particle injection and acceleration at collisionless shocks formed in energetic space objects, which harbour powerful outflows (supernova remnants, pulsar wind nebulae, active galactic nuclei, etc.). The number of spatial cells required to appropriately describe such an object, can be of order $10^{8}$. If at least two sorts of ions (say, hydrogen and helium) are taken into account, about $100 \mathrm{ppc}$ are need, i.e., ten billion particles are required. Being distributed among 100 nodes (2800 cores), it averages to about 350000 particles per MPI process. Hence, one step would take about $0.2 \mathrm{~s}$, which translates to about twenty-four hours for 500000 time steps. In fact, even such number of steps would not be enough to model a complex nonlinear configuration, because much smaller time steps may be required in that case. One may conclude, that most of the relevant astrophysical problems to be investigated with Maximus demand substantial computational power for relatively long periods of time (from weeks to months).

\section{Simulation of a Collisionless Shock in the Solar Wind}

The obvious way to verify numerical models is to confront their results to experimental data. In order to test the predictions of Maximus, we simulated distributions of accelerated ions in the Solar wind and compared them with the in-situ measurements made with the AMPTE/IRM interplanetary mission [17. The simulation was run with the following parameters, taken from the direct observational data: the upstream Mach number (in the downstream frame) $M_{A}=3.1$, the magnetic field inclination angle $\theta=10^{\circ}$, the ratio of thermal to magnetic pressure $\beta=0.04$. The standard Solar wind composition was considered: $90.4 \%$ $\mathrm{H}(+1)$ (by mass), $8.1 \% \mathrm{He}(+2)$, and about $1.5 \%$ of heavy ions, represented by $\mathrm{O}(+6)$. A shock was initialized in a rectangular box sized $10000 \times 400 l_{i}$ via 
the "rigid piston" method (reflection of a superalfvenic flow from a conducting wall). The simulated spectra of ions in the shock downstream are shown in Fig. 3 together with the experimental data. A good agreement of the model and the observations can be seen; the small differences may be due to variations of shock parameters. The acceleration Fermi process is illustrated in the supplementary video, where individual $\mathrm{H}(+1)$ (white circles) and $\mathrm{He}(+2)$ (green circles) ions positions are placed on the protons $x-E$ phase spaces, evolving with time. Both sorts of ions are reflected during the first shock encounter and subsequently gain energy by scattering upstream and downstream of the shock.

\section{Conclusions}

The 3d second-order accurate in time and space divergence-conserving hybrid code Maximus is presented. The code has been verified to keep energy and momentum conservation in modelled systems. It can be efficiently run on multi-core computers and clusters due to implementation of the MPI parallelization technology. For an illustrative case of the Solar wind, a good agreement of the modeling with the observational data of AMPTE/IRM has been demonstrated. Maximus can be effectively used to model nonlinear energetic processes in astrophysical plasmas on relevant spatial and time scales once substantial numerical resources are provided (hundreds of computational cores for time periods from weeks to months).

\section{Acknowledgments}

Some of the presented modeling was performed at the "Tornado" subsystem of the supercomputing center of St. Petersburg Polytechnic University, whose support is readily acknowledged.

\section{References}

1. Matthews Alan P. // J. Comput. Phys. Vol. 112. P. 102-116.

2. Lipatov A.S. The hybrid multiscale simulation technology. Berlin: Springer, 2002. $403 \mathrm{p}$.

3. Winske D., Omidi N. Hybrid Codes. Methods and applications. Computer Space Plasma Physics: Simulation Techniques and Softwares, 1993. P. 103-160.

4. Quest K.B., Journal of Geophysical Research. 93, 9649 (1988)

5. Giacalone J., Burgess D., Schwartz S.J., Ellison D.C., Bennett L., Journal of Geophysical Research. 102, 19789 (1997)

6. Burgess D., Möbius E., Scholer M., Space Science Reviews. 173, 5 (2012)

7. Caprioli D. and Spitkovsky A., ApJ. 783, Issue 2, 91 (2014)

8. Caprioli D. and Spitkovsky A., ApJ. 794, Issue 2, 46 (2014)

9. Caprioli D. and Spitkovsky A., ApJ. 794, Issue 2, 47 (2014)

10. Marcowith A., Bret A., Bykov A. et al, Reports on Progress in Physics. 79, Issue 4, id. 046901 (2016) 
11. Kropotina, Yu.A., Bykov, A.M., Krasilshchikov, A.M., Levenfish, K.P., JTePh, 60 (2015)

12. Kropotina, Yu.A., Bykov, A.M., Krasilshchikov, A.M., Levenfish, K.P., JTePh, 61 (2016)

13. Birdsall C.K., Langdon A.B. Plasma physics via computer simulation. Bristol: IOP Publishing, 1991. P. 373.

14. Hockney R.W. Measurements of Collision and Heating Times in a TwoDimensional

15. Gargaté L., Bingham R., Fonseca R.A. et al., Computer Physics Communication 176, 419 (2007)

16. Kunz M.W., Stone J.M., Bai X-N. // Journal of Computational Physics 259, 154 (2014).

17. Ellison, D.C., Moebius, E., Paschmann, G., ApJ 352, 376 (1990) Thermal Computer Plasma. Journal of computational physics 8, 19-44 (1971)

18. M. S. Rosin, A. A. Schekochihin, F. Rincon et al., MNRAS 413, 7 (2011)

19. Amano T., Higashimori K., Shirakawa K. // Journal of Computational Physics 275, 197 (2014).

20. Balsara D.S. // ApJS 151. P. 149-184, 2004

21. LeVeque R.J., Mikhalas D., Dorfi E.A., Muller E. Computational methods for Astrophysical Fluid Flow. Berlin: Springer, 1997. 481 p.

22. Balsara D.S. // ApJS 116. P. 119-131, 1998.

23. Florinsky V., Heerikhuisen J., Niemec J., Ernst A. // ApJ 826, 197 (2016)

24. Niemec J., Florinsky V., Heerikhuisen J., Nishikava K.-I. // ApJ 826, 198 (2016)

25. Kropotina, J.A., Bykov, A.M., Krasilshchikov, A.M., Levenfish, K.P. // JPCS, in press (2018). 


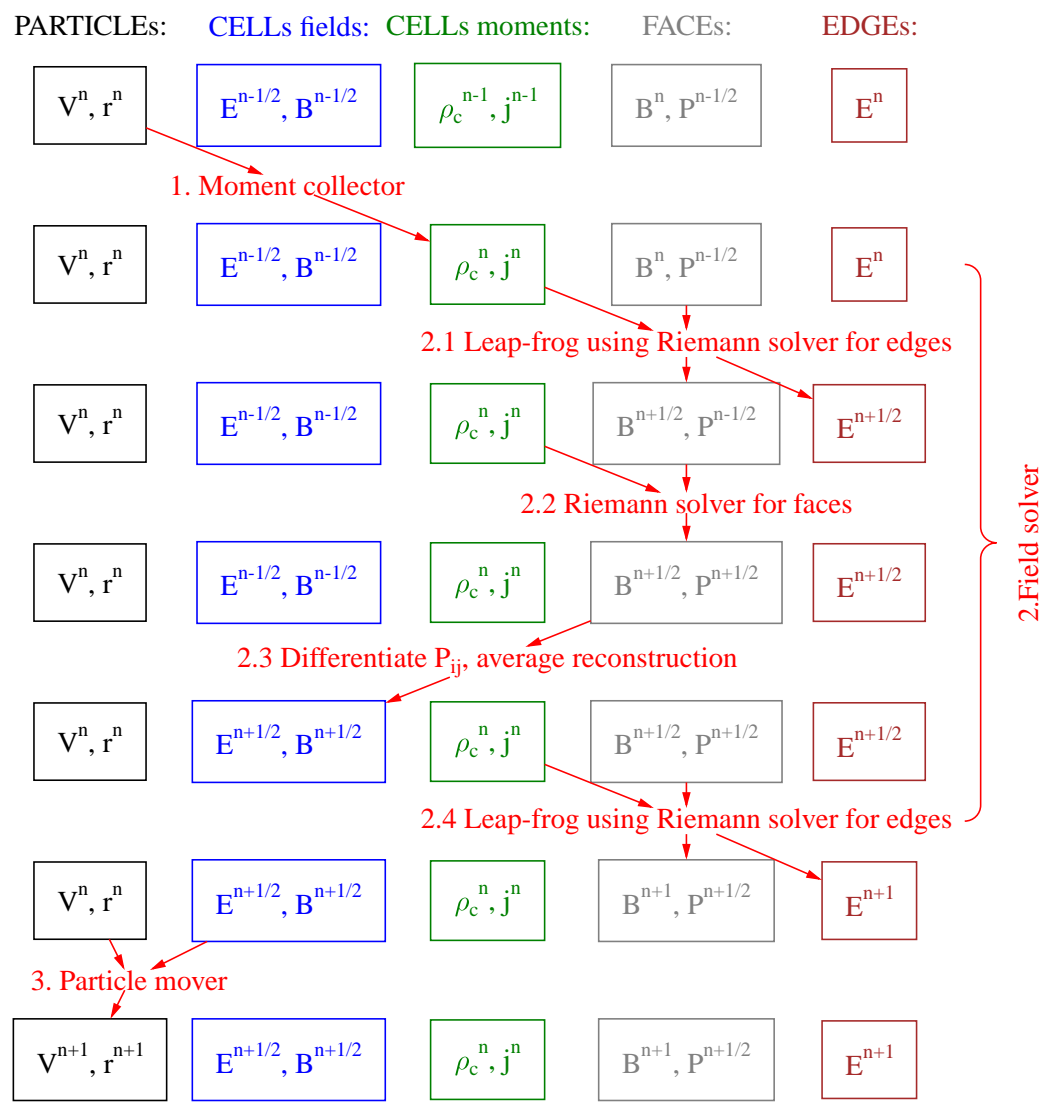

Fig. 1. A schematic illustration of the algorithm employed to update the variables from $t=t^{n}$ to $t=t^{n+1}$ (see Section 3 for a detailed description). The variables are shown as 5 color-coded categories. The red arrows indicate the substeps performed. 


\section{Simulation box}

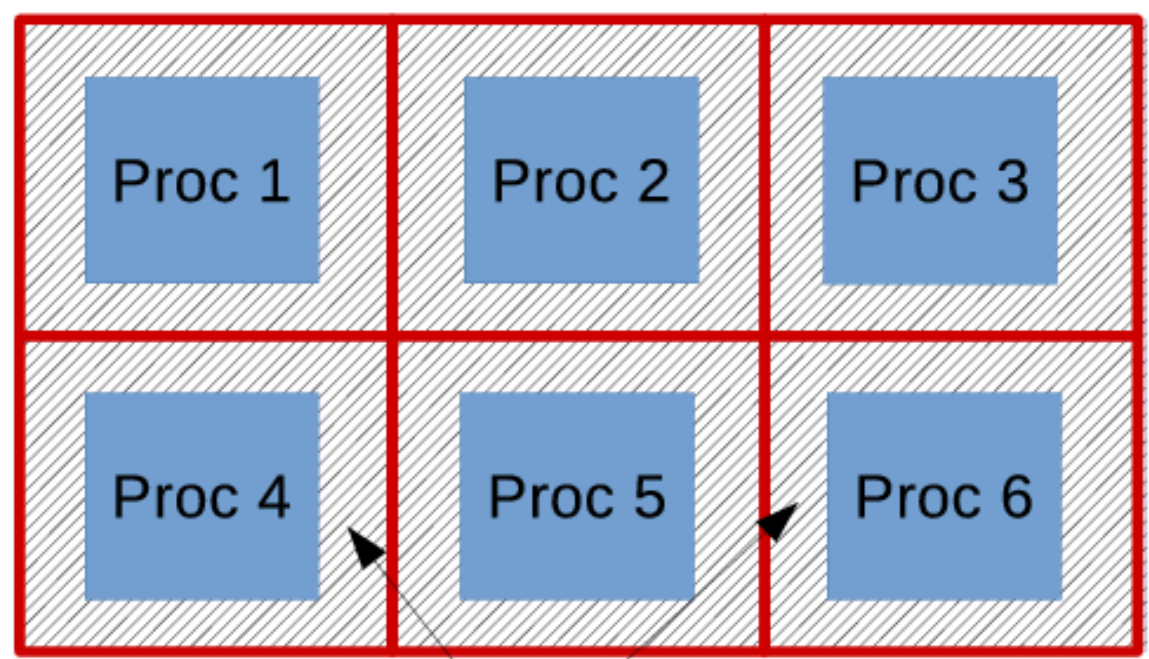

\section{Ghost cells}

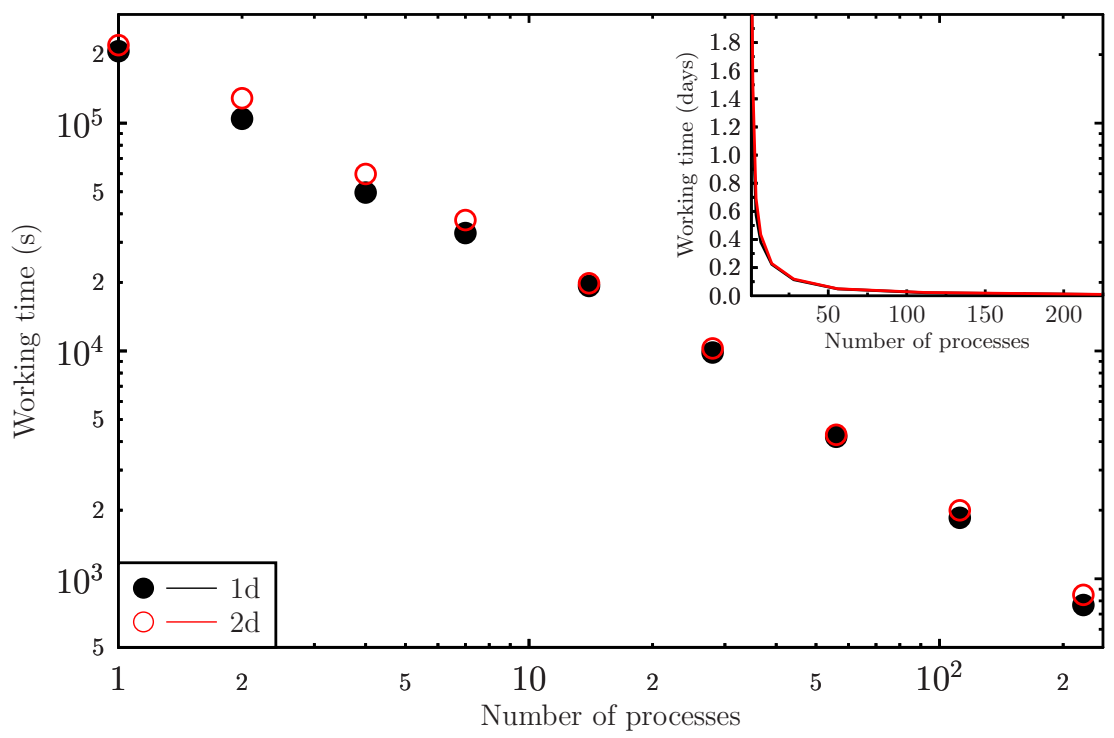

Fig. 2. Upper panel: Maximus parallelization scheme. The area allocated for each of the cores in the physical space is shown in blue, while ghost cells used for interconnection are hatched. Lower panel: Scalability of Maximus. Run time of the same tasks in $1 \mathrm{~d}$ and $2 \mathrm{~d}$ for different numbers of employed processor cores. The top-right inside panel shows the same time, measured in days, with linear scale. 


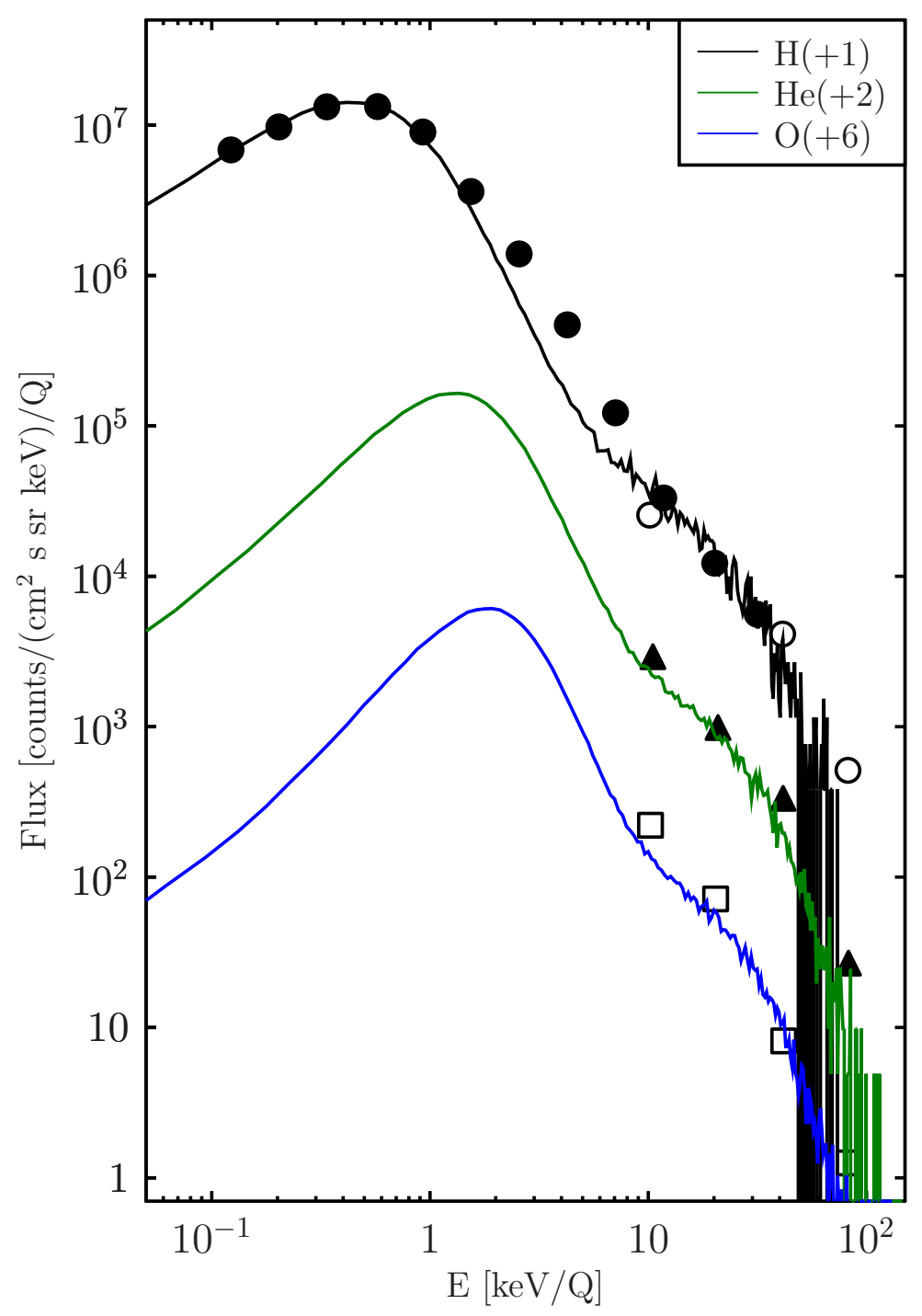

Fig. 3. Simulated energy distributions of ions downstream a shock in the Solar wind confronted with in-situ measurements of AMPTE/IRM interplanetary mission [17. 